\title{
Transport in the nonergodic extended phase of interacting quasiperiodic systems
}

\author{
Soumi Ghosh 1 , Jyotsna Gidugu, and Subroto Mukerjee \\ Department of Physics, Indian Institute of Science, Bangalore 560 012, India
}

(Received 19 July 2020; revised 18 October 2020; accepted 19 November 2020; published 23 December 2020)

\begin{abstract}
We study the transport properties and the spectral statistics of a one-dimensional closed quantum system of interacting spinless fermions in a quasiperiodic potential which produces a single-particle mobility edge in the absence of interaction. For such systems, it has been shown that the many-body eigenstates can be of three different kinds: extended and eigenstate thermalization hypothesis (ETH) obeying (thermal), localized and ETH violating (many body localized), and extended and ETH violating (nonergodic extended). Here we investigate the nonergodic extended phase from the point of view of level spacing statistics and charge transport. We calculate the dc conductivity and the low-frequency conductivity $\sigma(\omega)$ and show that both are consistent with subdiffusive transport. This is contrasted with diffusive transport in the thermal phase and blocked transport in the MBL phase.
\end{abstract}

DOI: $10.1103 /$ PhysRevB.102.224203

\section{INTRODUCTION:}

Anderson in his seminal paper (1958) [1] showed that sufficiently strong disorder can localize all the single-particle states in a disordered system. An extension of this phenomenon in the presence of interactions called many-body localization (MBL) was later argued to occur [2,3]. Since then, it has been a subject of immense interest, and there have been various numerical [4-6], phenomenological [7,8], and analytical [9] studies along with experimental verification [10,11]. Many-body localized systems are isolated interacting many-body systems which generically fail to thermalize $[3,5]$, violating the eigenstate thermalization hypothesis (ETH) [12-15]. As a consequence of the violation of ETH, the bipartite entanglement entropy of these systems in energy eigenstates is not equal to the thermal entropy. In fact, the entanglement in energy eigenstates follows an area law behavior in the MBL phase [16]. It has been argued that these systems have emergent conservation laws $[7,8,17,18]$ which prevent thermalization in a way similar to the nonergodic behavior seen in integrable systems [19-22]. As a consequence of localization there is no diffusion in the system. In the presence of interactions, for small enough disorder, generic many-body systems remain in a thermal phase. However, as the disorder strength is increased, for sufficiently large disorder, the system can undergo a thermal-MBL phase transition, which is a dynamical phase transition unlike the equilibrium phase transitions widely studied in physics. This thermal-MBL transition has also been shown to exist in the presence of a quasiperiodic potential instead of true random disorder $[10,16]$.

The simplest quasiperiodic potential is the Aubry-André potential [23] $\left[h_{i}=h \cos (2 \pi \alpha i+\phi)\right]$, in the presence of which the noninteracting system has extended or localized single-particle states depending on the potential strength $h$. For $h<2 t$ all the single-particle energy eigenstates are extended, and for $h>2 t$ all the energy eigenstates are localized, where $t$ is the hopping parameter. Upon switching on interactions, this delocalized to localized transition transforms into a thermal-MBL transition where for $h<h_{c}$ all typical mid-spectrum states are thermal and for $h>h_{c}$ the typical states are many body localized [16,24].

However, there are other quasiperiodic potentials [25-29] which have single-particle mobility edges (SPMEs) in the absence of interactions up to some critical potential strength, and the SPME is defined by a critical energy $E_{c}$ that separates the extended and localized eigenstates of the single-particle Hamiltonian. In fact, single-particle mobility edges arise generically for quasiperiodic potentials. The Aubry-André model is, in a sense, fine-tuned, due to which it possesses an energy-independent duality which does not allow the existence of a mobility edge.

In the presence of interactions these models also undergo transitions from thermal to athermal phases [30,31]. However, it has been argued that these models are different from those without single-particle mobility edges in that they possess a nonergodic extended phase at the intermediate potential strengths. In this phase, there are states in the energy spectrum that are nonergodic in the sense that they violate the ETH but are also extended since they possess a volume law satisfying entanglement entropy [32,33]. It has been argued [32] that this phase can be thought of as arising from the many-body states of the noninteracting model which are Slater determinants with both extended and localized states upon turning on interactions "adiabatically." This kind of nonergodic state was also described in Josephson junction arrays [34], where it was shown that there is a phase transition as a function of temperature where the metallic phase at low temperature and the many-body localizing phase at higher temperatures were separated by a "bad-metal" phase where the system is nonergodic but conducting. 
The transport properties of one-dimensional systems are very interesting as there can be rich varieties of transport in one-dimensional systems depending on the underlying potential in both the interacting and noninteracting regimes [35-44]. In this paper we investigate the transport properties of the nonergodic extended phase in systems with a singleparticle mobility edge. Using numerical exact diagonalization, we obtain the energy level spacing statistics and the dc and ac conductivities, in terms of which we characterize the phase. Here we consider spinless fermions in one dimension described by the Hamiltonian

$$
H=-t \sum_{i}\left(c_{i+1}^{\dagger} c_{i}+\text { H.c. }\right)+\sum_{i} h_{i} n_{i}+V \sum_{i} n_{i} n_{i+1},
$$

where $t$ is the hopping parameter, $V$ is the nearest-neighbor interaction strength, and the on-site potential $h_{i}$ is chosen to be the quasiperiodic potential $h_{i}=h \frac{\cos (2 \pi \alpha i+\phi)}{1-\beta \cos (2 \pi \alpha i+\phi)}$, which has a mobility edge [27] at $\beta E_{c}=2 \operatorname{sgn}(h)(|t|-|h| / 2)$, separating the single-particle extended states from the localized ones. We calculate the level statistics and the transport properties numerically using exact diagonalization for different filling fractions $(v=1 / 2,1 / 4,1 / 6)$. For better statistics we average all the quantities over a randomly chosen offset angle $\phi \in$ $[0,2 \pi)$ and apply periodic boundary conditions in all our calculations. We calculate the variation of average level spacing ratio as a function of energy density to locate a critical energy density separating the energy eigenstates with Poisson energy level spacing statistics from the states with the energy level spacing following a Gaussian orthogonal ensemble (GOE) distribution. We also examine the violation of ETH and obtain the entanglement scaling as a function of energy density at these different filling fractions to locate transitions from a nonergodic to ergodic phase and localized to delocalized phase, respectively, in the energy spectrum [32]. We obtain the critical values of energy density at which these transitions occur.

The disordered $X X Z$ model showed a diffusive transport deep in the thermal phase in the presence of small disorder when subdiffusive transport was reported [40,41] in the thermal region near the thermal-MBL transition. It has been shown that the ac conductivity vanishes at low frequency as a power law $\sigma(\omega) \sim \omega^{\alpha}$, where the parameter $\alpha \rightarrow 1$ as the localized phase is approached, $\alpha \rightarrow 0$ in the diffusive phase, and for the subdiffusive phase it has a value $0<\alpha<1$. The subdiffusive phase in the random $X X Z$ model is associated with the Griffiths effects where rare local regions of strong disorder in a random one-dimensional system act as insulating regions and restrict transport in the system [40].

In this paper we show that in the presence of a nonergodic extended phase, the ac conductivity vanishes at low frequency following a power law $\sim \omega^{\alpha}$, where $\alpha$ has a value between 0 and 1 for different filling fractions, indicating subdiffusive behavior, and that the dc conductivity decreases with the increasing system sizes but decays slower than the exponential decay expected in the MBL phase [43], indicating subdiffusive transport. We also calculate the individual contributions to the dc conductivity from the three different phases in the energy spectrum, namely, the many-body localized, nonergodic extended, and ergodic phases, to extract the information about the scaling of the same with increasing system sizes and find that the contribution from the nonergodic extended phase decays more slowly than the contribution from the many-body localized phase, while the contribution from the ergodic phase hardly decays at all with the increasing system sizes.

\section{CONDUCTIVITY CALCULATIONS}

To study the charge transport within the system subject to periodic boundary conditions we use the Kubo formula for the conductivity $\sigma(\omega)$,

$$
\sigma(\omega)=\frac{\pi}{L} \frac{1-e^{\beta \omega}}{\omega} \sum_{m, n} e^{-\beta E_{m}}|\langle m|J| n\rangle|^{2} \delta\left(E_{n}-E_{m}-\omega\right),
$$

where $J=-i t \sum_{i}\left(c_{i+1}^{\dagger} c_{i}-c_{i}^{\dagger} c_{i+1}\right)$ is the total current operator and $|n\rangle$ and $|m\rangle$ are many-body eigenstates of the system.

In the study of many-body localization, localization properties of the closed quantum systems are typically studied at infinite temperature. This is because the many-body density of states is sharply peaked in the middle of the spectrum for a sufficiently large system. Hence, if all energy eigenstates are chosen with equal weight, which corresponds to putting the system in contact with an infinite-temperature $(\beta \rightarrow 0)$ bath any quantity calculated by averaging over the whole energy spectrum gives the typical mid-spectrum value of that quantity. The most convenient way to calculate the conductivity is to use the Kubo formula, which has temperature as a parameter. Thus, to obtain the contributions to the conductivity from all the energy eigenstates in an unbiased manner, the temperature is set to infinity for our calculations. At infinite temperature, the Kubo formula reduces to

$$
T \sigma(\omega)=\frac{\pi}{Z L} \sum_{m, n}|\langle m|J| n\rangle|^{2} \delta\left(E_{n}-E_{m}-\omega\right),
$$

where $Z$ is the partition function. The many-body energy spectrum is discrete for finite-sized systems and approaches a continuous spectrum in the thermodynamic limit. The discreteness of the energy spectrum results in the conductivity being a sum of discrete $\delta$ functions rather than the smooth function of $\omega$ expected in the thermodynamic limit. Thus, for a finite-sized system, the $\delta$ functions are approximated by a Lorentzian function of the form $\delta(E)=\frac{1}{\pi} \frac{\eta}{\eta^{2}+E^{2}}$, where the width of the Lorentzian peak $2 \eta$ is chosen such that $\eta \ll \Delta$. This allows one to obtain a smooth form for $\sigma(\omega)$. Here $\Delta$ is the mean level spacing of the many-body spectrum.

\section{A. Low-frequency conductivity}

Here we calculate the low-frequency conductivity $\sigma(\omega) \sim$ $\omega^{\alpha}$ and obtain the exponent $\alpha$ for different values of the filling fraction, $v=\frac{1}{2}, \frac{1}{4}, \frac{1}{6}$, for a typical set of parameters $h=0.6, \beta=-0.8, V=1.0, t=1.0$. The conductivity $\sigma(\omega)$ is calculated by averaging over 1024 randomly chosen offset $\phi$ 's for all the filling fractions.

It is known that [45] near the MBL transition $\alpha \rightarrow 1$, and it remains between 1 and 2 in the MBL phase. In the thermal phase the conductivity is expected to be diffusive, which means that $\sigma(\omega)$ at small $\omega$ should tend to $\sigma_{\mathrm{dc}} \neq 0$ and the exponent $\alpha=0$. In [40], a subdiffusive phase with $0<\alpha<1$ 

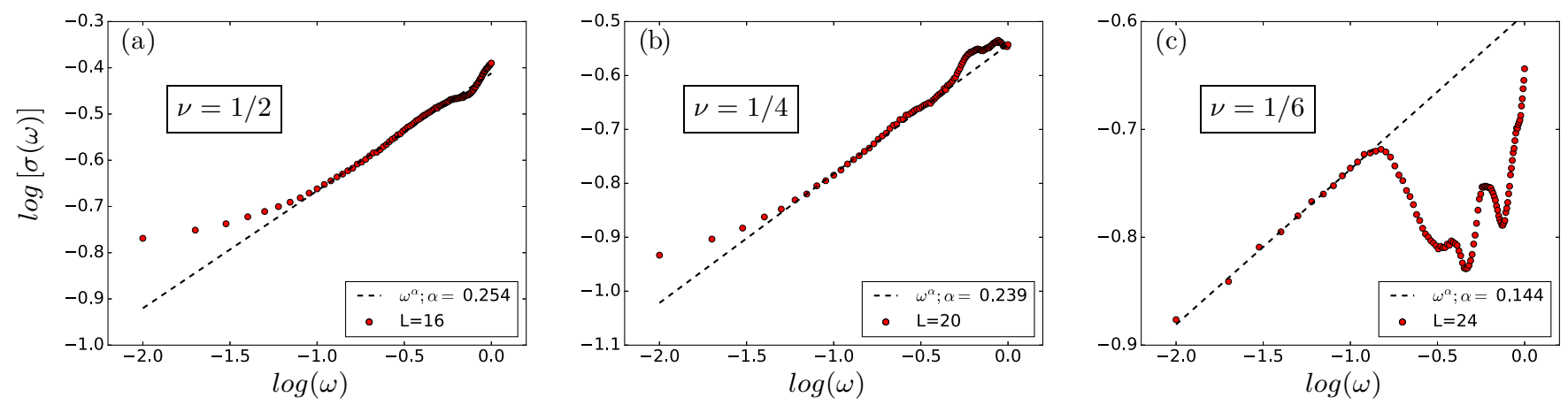

FIG. 1. Log-log plot showing the variation of $\sigma(\omega)$ as a function of $\omega$ for different filling fractions $v=\frac{1}{2}$ (a), $\frac{1}{4}$ (b), $\frac{1}{6}$ (c) at system sizes $L=16,20$, and 24, respectively. The dashed lines are a fit of the power law $\sigma(\omega) \sim \omega^{\alpha}$ at frequencies well below the hopping parameter $t$. The fitted parameter $\alpha$ belongs to the subdiffusive range for all filling fractions. The width $\eta$ of the Lorentzian (broadening of the $\delta$ function in the Kubo formula) is chosen to be $0.1 \Delta$, where $\Delta$ is the mean level spacing.

was reported, signaling the onset of Griffiths effects in a system with random disorder.

Figure 1 shows the variation of $\sigma(\omega)$ for small values of $\omega<t$. We fit a power law $\sim \omega^{\alpha}$ for $\omega>\Delta$, with $\Delta$ being the mean level spacing. For all the filling fractions $v$ we see a value $\alpha$ corresponding to the subdiffusive transport. We choose this region to do the power law fitting because of its lower susceptibility to the finite-size effects due to the level repulsion in the system. However, up to the finite-size effects, the exponent obtained by fitting the power law at the lowest $\omega$ in Fig. 1 still shows subdiffusive behavior. We note that there are no Griffiths regions in our systems since the on-site potential is completely deterministic. We thus attribute the subdiffusive transport to the presence of the nonergodic extended phase in these systems, as discussed further in the following sections.

Choice of $\eta$. Figure 2 shows the variation of the $\sigma(\omega)$ versus $\omega$ curve as we change the width of the Lorentzian $2 \eta$ for the system sizes $L=12,12,18$ at fillings $v=\frac{1}{2}, \frac{1}{4}, \frac{1}{6}$, respectively. Here the sample sizes chosen are 4800, 6400, and 4800, respectively, for the three filling fractions $(1 / 2,1 / 4,1 / 6)$. As we see, only the $v=\frac{1}{4}$ case has any significant variation as $\eta$ is changed from $0.001 \Delta$ to $\Delta$, where $\Delta$ is the mean level spacing. This can be understood as follows: The systems we are studying display level repulsion in parts of their spectrum (see Sec. III). The low-energy density part displays no level repulsion, while the rest of the spectrum (which we call the high-energy density part here) does. At very low frequencies (less than the level spacing $\Delta$ ), where the variation with $\eta$ is most pronounced, the contribution to $\sigma(\omega)$ in Eq. (1) comes mainly from the high-energy density part of the spectrum since the low-energy density part has a large number of localized states, which do not contribute significantly. Further, the contribution to $\sigma(\omega)$ from the matrix elements of the current between states, one of which is in the low-energy density part and the other of which is in the high-energy density part, is also not significant since $\omega$ is small. Now, since $\omega<\Delta$ and level repulsion is operative, the number of pairs of states with energy difference $\omega$ is not very large. Broadening each energy level by a factor $\eta$ thus significantly changes the effective number of such pairs, giving rise to a noticeable change in the value of $\sigma(\omega)$. At higher frequencies $\omega>\Delta$, since level repulsion is not strongly operative anymore, the number of energy pairs contributing to $\sigma(\omega)$ does not change much due to broadening and thus neither does $\sigma(\omega)$. Now, for $v=\frac{1}{4}$ at $L=12, p=3$, the mean level spacing $\Delta \sim 0.05$, while for $v=\frac{1}{2}$ and $\frac{1}{6}, \Delta \sim 0.01$ at $L=12$ and 18 , respectively. Thus, the lowest frequencies in Fig. 2 already satisfy $\omega \gtrsim \Delta$ for $v=\frac{1}{2}$ and $v=\frac{1}{6}$, and there is no appreciable dependence on $\eta$. On the other hand, for $v=\frac{1}{4}$, the smallest values of $\omega$ in Fig. 2 correspond to $\omega<\Delta$, and there is an appreciable dependence of $\sigma(\omega)$ at these values on $\eta$. In fact, it can also
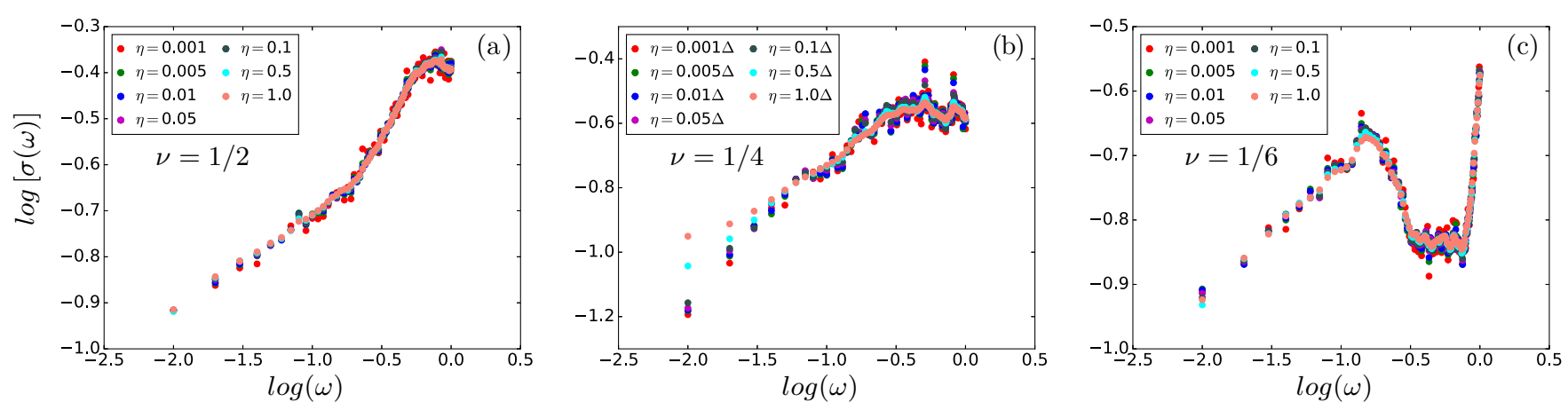

FIG. 2. The variation $\sigma(\omega)$ vs $\omega$ with the change in broadening width $\eta$ of the Lorentzian for different filling fractions $v=\frac{1}{2}$ (a), $\frac{1}{4}$ (b), $\frac{1}{6}$ (c) at system sizes $L=12,12,18$, respectively. Here $\eta$ is chosen to be some fraction $f$ of the mean level spacing $\Delta$, where $f$ is changed from 0.001 to 1.0 for all three cases. 


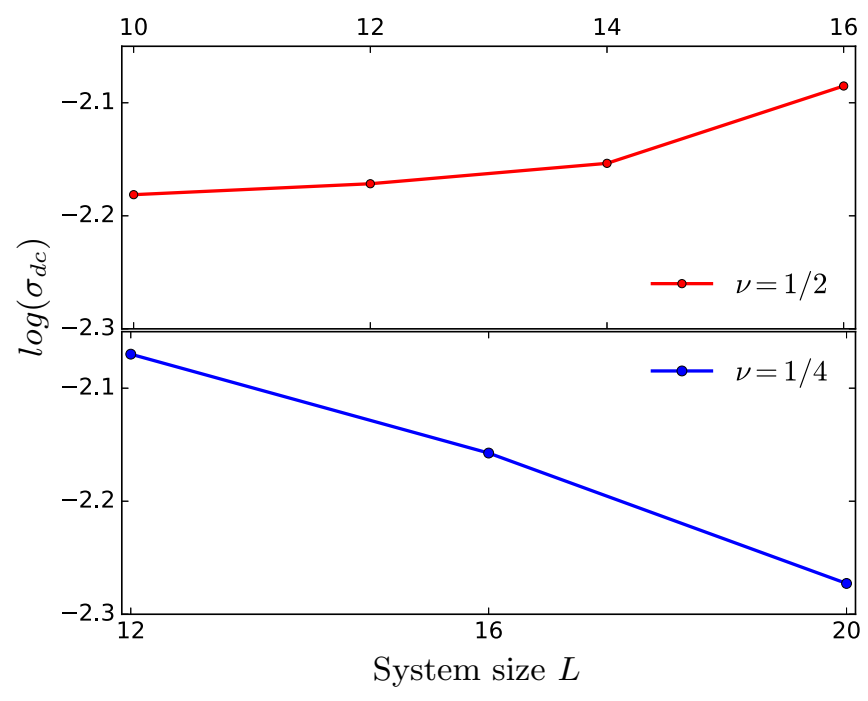

FIG. 3. Variation of the dc conductivity $\sigma_{\mathrm{dc}}$ with increasing system sizes at filling fractions $v=1 / 2$ (red line in the top panel) and $v=1 / 4$ (blue line in the bottom panel). The width $\eta$ of the Lorentzian is chosen to be $0.01 \Delta$, where $\Delta$ is the mean level spacing.

be seen from Fig. 2(b) for $v=1 / 4$ that the dependence on $\eta$ starts disappearing at $\log _{10} \omega \approx-1.3$, which indeed corresponds to $\omega \approx \Delta$. It is also for this reason that increasing the system size reduces the dependence on $\eta$ because it reduces the value of $\Delta$.

\section{B. de conductivity}

We also calculate the dc conductivity by taking the limit $\omega \rightarrow 0$ in Eq. (1):

$$
\lim _{\omega \rightarrow 0} T \sigma(\omega)=\frac{\pi}{Z L} \sum_{m, n}|\langle m|J| n\rangle|^{2} \delta\left(E_{n}-E_{m}\right) .
$$

A diffusive system must have a nonzero dc conductivity, while many-body localized systems are expected to have no transport at all, which implies zero dc conductivity. However, finite-size systems will have some nonzero value of the dc conductivity, and for MBL systems this de conductivity decays exponentially with increasing system sizes, implying no transport in the thermodynamic limit $[38,39,43]$.

Here we calculate the dc conductivity for different filling fractions $v=\frac{1}{2}, \frac{1}{4}$ at $h=0.6, \beta=-0.8, V=1.0, t=$ 1.0 and look for the system size scaling of the same. The sample sizes chosen are $108000,108000,18000$, and 1800 for $L=10,12,14,16$, respectively, at $v=1 / 2$ and 108000 , 108000 , and 900 for $L=12,16,20$, respectively, at $v=1 / 4$. Since for $v=1 / 6$ we have only two system sizes accessible $(L=18,24)$ through exact diagonalization, we do not investigate the scaling of the dc conductivity in this case.

Figure 3 shows the variation of $\log _{10}\left(\sigma_{\mathrm{dc}}\right)$ with increasing system sizes at different filling fractions $v$. While for $v=1 / 2$ (Fig. 3, top panel) the $\sigma_{\mathrm{dc}}$ remains roughly the same order with increasing system size, for $v=1 / 4$ (Fig. 3, bottom panel) we see decay with increasing system size, which is slower than that in the MBL phase, where the decay is expected to be exponential [43]. The behavior for $v=1 / 2$ appears to be similar to diffusive behavior, as seen numerically in other systems
[43]. However, it is important to note that the contributions to $\sigma_{\mathrm{dc}}$ come from three different regions in the energy spectrum (thermal, nonergodic extended, and MBL), each of which contributes a different type of behavior (diffusive, subdiffusive, and localized). The observed system-size dependence is thus a function of the relative sizes of the three regions in the energy spectrum. For $v=1 / 2$, diffusive behavior appears to dominate, while for $v=1 / 4$, subdiffusive behavior seems to be dominant. This behavior is further investigated in Sec. IV, where we calculate the contribution of the three different energy regions to $\sigma_{\mathrm{dc}}$ separately.

Choice of width $\eta$. Since we are working in the regime $\omega \rightarrow 0$, changing the width $\eta$ of the Lorentzian corresponding to $\delta\left(E_{n}-E_{m}\right)$ implies changing the number of energy pairs $\left(E_{n}, E_{m}\right)$ contributing to $\sigma_{\mathrm{dc}}$. Therefore, decreasing $\eta$ leads to a decrease in the number of energy pairs contributing to $\sigma_{\mathrm{dc}}$ and hence a decrease in $\sigma_{\mathrm{dc}}$. However, since $\eta$ is chosen to be a fraction of the corresponding mean level spacing $\Delta$, the nature of the variation of $\sigma_{\mathrm{dc}}$ with $L$ does not change. Only the absolute value of the dc conductivity changes with $\eta$, as seen in Fig. 4. The sample sizes chosen for this calculation are (102 400, 6400, 128) for $L=12,14,16$, respectively, in the case of $v=1 / 2$ and $(10240,10240,128)$ for $L=12,16,20$, respectively, for filling fraction $v=1 / 4$.

\section{LEVEL SPACING STATISTICS, ETH VIOLATION, AND ENTANGLEMENT ENTROPY}

To identify the nonergodic extended phase we employ different diagnostics which differentiate between nonergodic and ergodic phases and extended and localized phases. The parameters of the Hamiltonian have the same values as those in the conductivity calculations.

a. Level Spacing statistics. The level spacing statistics shows the presence or absence of repulsion in the energy spectrum. Level repulsion is generally associated with the ergodicity of the system, and its absence is associated with the nonergodicity. It was argued that for a localized system, two eigenstates with similar energies are far apart in terms of overlap and hence do not experience any level repulsion. As a result, successive energy gaps become Poisson distributed [3]. On the other hand, in the ergodic phase, there is level repulsion, and the level spacing statistics is that of a random matrix theory, specifically, the GOE for the systems we consider. To identify the two different types of behavior, we calculate the average of the ratio of successive energy gaps $r_{n}=\frac{\min \left\{\delta_{n}, \delta_{n+1}\right\}}{\max \left\{\delta_{n}, \delta_{n+1}\right\}}$, where $\delta_{n}=E_{n+1}-E_{n}$ is the energy gap between the $n$th and $(n+1)$ th energy levels. It is known that the average value of this ratio is $\simeq 0.529$ for the GOE, while for a Poisson distribution it is $\simeq 0.386$. Thus, the average level spacing ratio can be used to locate the ergodic and nonergodic phases. Here we divide the whole many-body energy spectrum into equal bins and calculate the average level spacing ratio for the successive eigenstates belonging to the same energy bin. While calculating the level spacing ratio, we average over 10000,5000 , and 200 realizations of the random phase $\phi$ for system sizes $L=12,14,16$, respectively, for $v=1 / 2$; 10000,4800 , and 96 realizations of the random phase $\phi$ for system sizes $L=12,16,20$, respectively, for $v=1 / 4$; and 

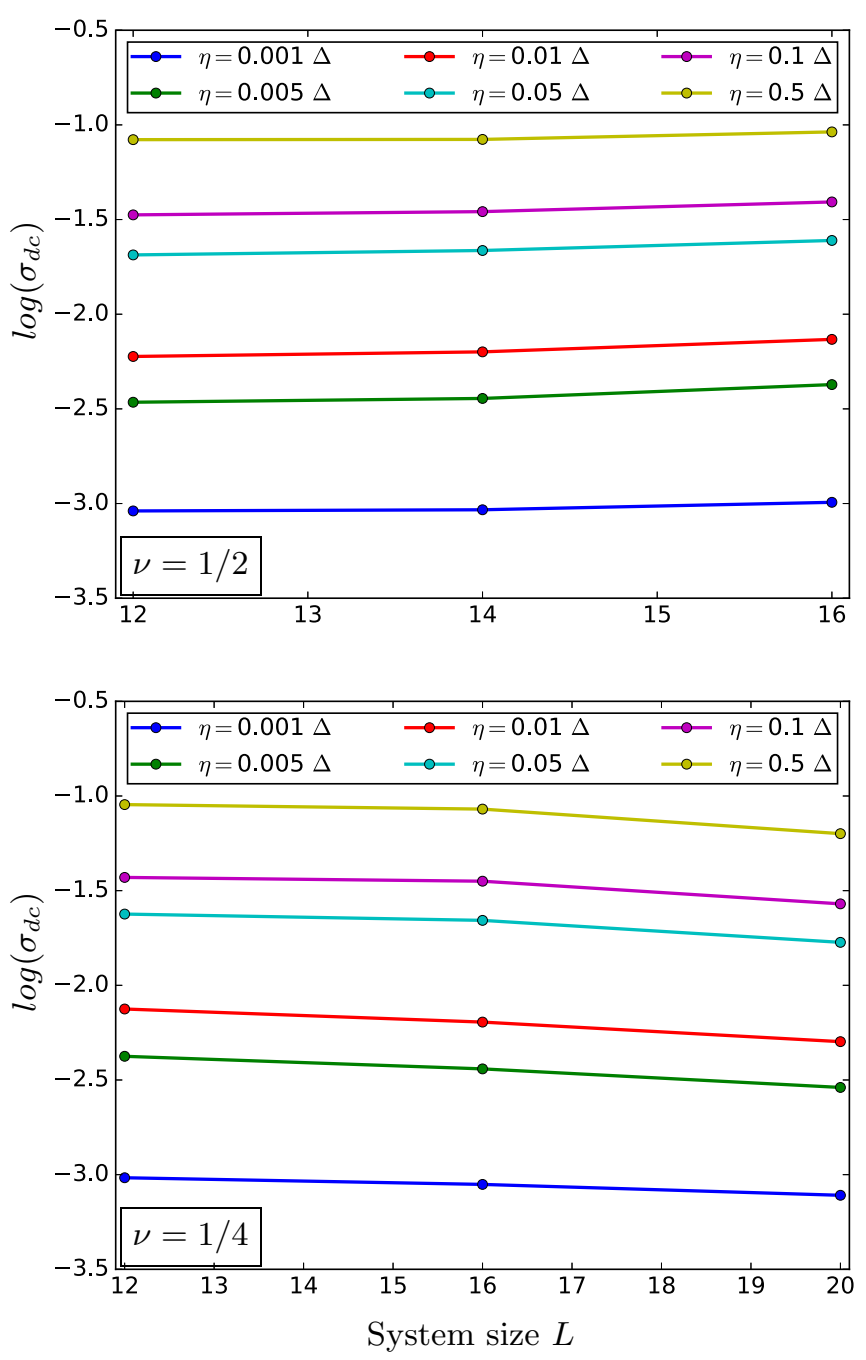

FIG. 4. Variation of dc conductivity $\sigma_{\mathrm{dc}}$ vs system size for different choices of width $\eta$ of the Lorentzian at filling fractions, $v=1 / 2$ (top) and $v=1 / 4$ (bottom). Again, $\eta$ is chosen as fraction $f$ of the mean level spacing $\Delta$, where $f$ is changed from 0.001 to 1.0 for both cases.

9600 and 100 realizations of the random phase $\phi$ for system sizes $L=18,24$, respectively, for $v=1 / 6$.

As seen in Figs. 5(a)-5(c) there is a value of energy $E_{r}$ (blue dashed line) for all filling fractions up to which all the energy eigenstates display Poisson level spacing statistics, implying the absence of level repulsion in the systems. Above this value of the energy density all the energy eigenstates display GOE statistics, implying ergodicity in the system.

b. ETH violation. The eigenstate thermalization hypothesis [12-14] states that in an ergodic system the expectation value of a local operator in any many-body eigenstate $|n\rangle$ corresponds to the thermal expectation value at the corresponding energy $E_{n}$. In other words, any single energy eigenstate gives the same expectation value of a local operator as the one calculated by averaging over nearby energy eigenstates, i.e., the thermal expectation value corresponding to the microcanonical ensemble. The fluctuation of the expectation value in nearby energy eigenstates is exponentially small in the system size. Therefore, if we define an observable such as the total number of particles in one half of the system,

$$
O\left(E_{n}\right)=\sum_{j=1}^{N / 2}\left\langle n\left|n_{j}\right| n\right\rangle,
$$

then $O\left(E_{n}\right)$ should be similar for nearby many-body energy eigenstates if the system is ergodic [32], and a fluctuation of the values of $O\left(E_{n}\right)$ should imply nonergodicity.

However, for finite-size systems there are always finite fluctuations in $O\left(E_{n}\right)$ between adjacent energy eigenstates for both ergodic and nonergodic phases. Hence, we distinguish between the two phases by looking at the fluctuation in the quantity $O\left(E_{n}\right)$ among nearby energy eigenstates; in an ergodic phase where ETH holds, this is exponentially small in the system size, whereas in a nonergodic phase, it is of the order of 1 for sufficiently large system sizes.

We divide the many-body spectrum into bins and calculate the variance of $O\left(E_{n}\right)$ within each bin. The quantity is averaged over the same number of samples for the offset $\phi$ as in the level spacing ratio calculation. Figures 5(d)-5(f) show the variance of $O\left(E_{n}\right)$ within nearby energies as a function of energy density. The value of $\operatorname{var}[O]$ does not decrease (and, in fact, appears to increase) with system size up to an energy density equal to $E_{O}$, indicating a nonergodic phase for all filling fractions up to that energy. This type of behavior has been seen in previous numerical studies [32]. Since there is no physical reason for why the variance should increase with system size, we expect it to eventually saturate for sufficiently large (but currently numerically inaccessible) system sizes. For larger energy densities, var[O] gets suppressed with increasing system size, indicating an ergodic phase. We also note that $E_{r} \simeq E_{O}$.

The finite-size effects for $v=1 / 4$ and $v=1 / 6$ in the calculation of $\langle r\rangle$ and $\operatorname{var}[O]$ are more pronounced than for $v=1 / 2$. The location of the transition from the nonergodic to ergodic phase obtained using $\operatorname{var}[O]$ is the lowest value of $\epsilon$ at which $\operatorname{var}[O]$ starts decreasing with increasing system size. This is what we expect to see in the ergodic phase. For $\langle r\rangle$, the location of the transition for $v=1 / 2$ is determined by identifying where its value reaches the Wigner-Dyson value, while for $\operatorname{var}[O]$, it is determined from the location at which it decreases with increasing system size. It can be seen than the two transitions are concurrent for $v=1 / 2$. For $v=1 / 4$ and $v=1 / 6$, the transition from $\operatorname{var}[O]$ can still be located using the same criterion as for $v=1 / 2$, but it becomes difficult to do so for $\langle r\rangle$ because of the far more pronounced finite-size effects. Instead, for these two values of filling, we mark the value of $\langle r\rangle$ at the transition value of $\epsilon$ as determined from $\operatorname{var}[O]$ and note that it is "sufficiently" far away from the Poissonian value. If we use the energy density value at which $\langle r\rangle$ for the largest system in the two cases reaches the WignerDyson value as an additional bound, we obtain a range of $\epsilon=-0.39$ to -0.24 for $\nu=1 / 4$ and $\epsilon=-0.2$ to -0.12 for $v=1 / 6$ for the transition. Note that in both cases, the transition is sufficiently distant from the localization-delocalization transition (discussed below), which occurs at $\epsilon \approx-0.6$ for $\nu=1 / 4$ and $\epsilon \approx-0.42$ for $v=1 / 6$. Showing this distinct separation of the two transitions is one of the main points of the plots. 

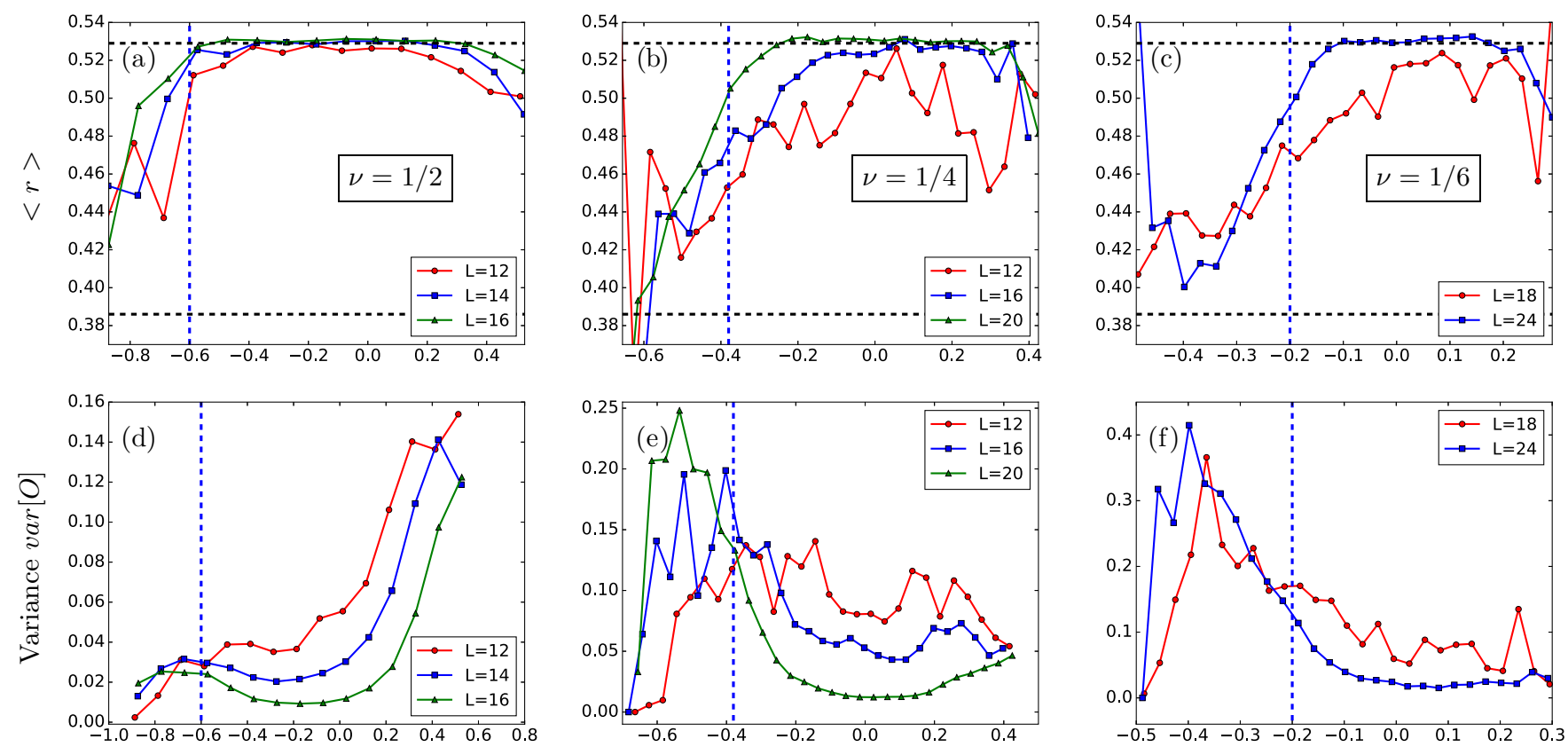

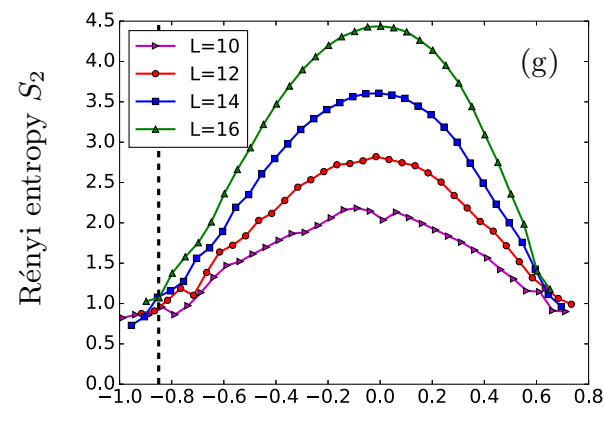

Energy density $\varepsilon$

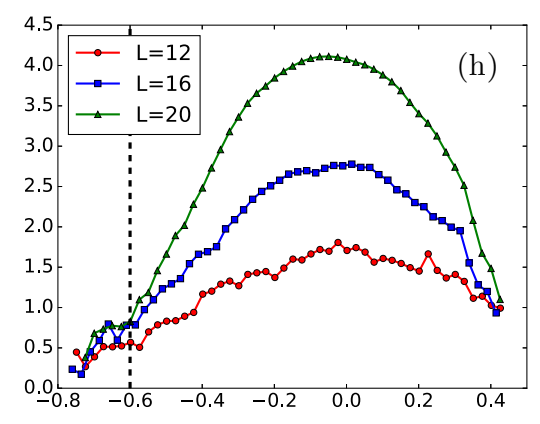

Energy density $\varepsilon$

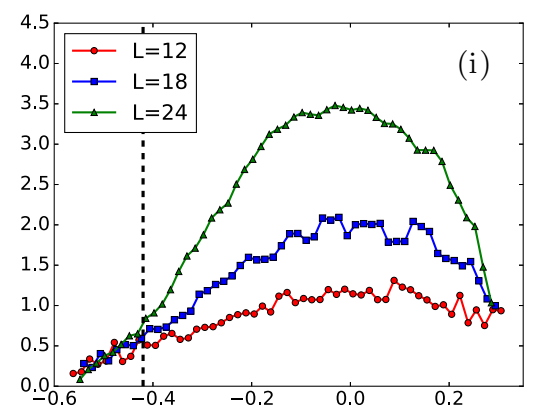

Energy density $\varepsilon$

FIG. 5. The variation of the average level spacing ratio $r_{n}$ as a function of energy density for different filling fractions $v$ : (a) $\frac{1}{2}$, (b) $\frac{1}{4}$, (c) $\frac{1}{6}$. The location of transition point $E_{r}$ is indicated by the blue dashed lines (as discussed in Sec. III A). The horizontal black dashed lines in (a)-(c) depict the average value of the level spacing ratio for GOE $(\simeq 0.529)$ and Poisson $(\simeq 0.386)$ distributions. The variance of the local observable $O$ as a function of energy density is shown for different filling fractions $v$ : (d) $\frac{1}{2}$, (e) $\frac{1}{4}$, (f) $\frac{1}{6}$. The location of the ergodic-nonergodic transition $E_{0}$ is indicated by the vertical dashed lines (as discussed in Sec. III B). The bipartite Rény entropy is shown for different filling fractions $v$ : (g) $\frac{1}{2}$, (h) $\frac{1}{4}$, (i) $\frac{1}{6}$. The location of the localization-delocalization transition $E_{s}$ is indicated by the black dashed line (as discussed in Sec. III C).

c. Entanglement entropy. To study the localizationdelocalization transition, here we study the bipartite Rényi entropy between the two halves of the system $[32,46]$. For that we divide a lattice of size $L$ into two halves, $A$ and $B$ of size $L / 2$, and compute the Rényi entropy $S_{2}$ using

$$
S_{2}(L / 2)=-\log _{10}\left(\operatorname{Tr} \rho_{A}^{2}\right)
$$

where $\rho_{A}$ is the reduced density matrix obtained from the density matrix $\rho=\left|\Psi_{E}\right\rangle\left\langle\Psi_{E}\right|$ by taking a partial trace over subsystem $B$. Here $\Psi_{E}$ is the many-body eigenstate corresponding to many-body energy $E$.

Volume law behavior of the entanglement entropy implies delocalized states, while area law behavior implies localization in the system. Here we calculate the entanglement entropy as a function of energy density by binning the many-body energy spectrum and calculating the average Rényi entropy for each bin. While calculating the entropy, we average over 100,100,100, and 10 realizations of the random phase $\phi$ for system sizes $L=10,12,14,16$, respectively, for $v=1 / 2$; and 50, 20, and 10 realizations of the random phase $\phi$ for system sizes $L=12,16,20$, respectively, for $v=1 / 4$; and 1000,100 , and 15 realizations of the random phase $\phi$ for system sizes $L=12,18,24$, respectively, for $v=1 / 6$.

Figures 5(g)-5(i) show the variation of Rényi entropy $S_{2}$ as a function of energy density. There is an energy density value $E_{S}$ (marked by the dashed line) which separates the manybody energy eigenstates following area law behavior to the left of $E_{S}$ from the volume law following eigenstates on the right.

We see that the energy density $E_{r}$ that separates the energy levels following a Poissonian level spacing distribution from the ones following GOE is almost equal to the energy density $E_{O}$ separating the energy eigenstates violating ETH from those that obey ETH. We also see the energy density $E_{S}$ that separates the localized eigenstates from delocalized eigenstates is less than $E_{r} \simeq E_{O}$. Therefore, we have three different 


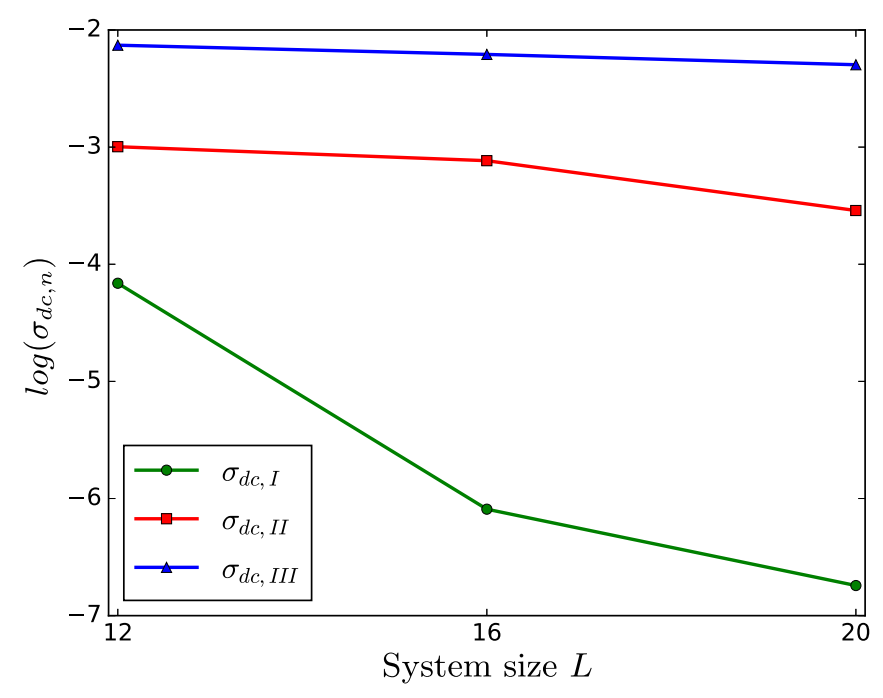

FIG. 6. Variation of contributions of the three regions to $\sigma_{\mathrm{dc}}$ $\left(\sigma_{\mathrm{dc}, I}, \sigma_{\mathrm{dc}, I I}\right.$, and $\left.\sigma_{\mathrm{dc}, I I}\right)$ with increasing system sizes are shown for filling fraction $v=\frac{1}{4}$. The width $\eta$ is chosen to be $0.01 \Delta$, where $\Delta$ is the mean level spacing. The region I corresponds to the nonergodic localized states, II corresponds to the nonergodic extended states and III corresponds to the ergodic extended states.

regions for all three filling fractions considered: localized and nonergodic (energy density less than $E_{S}$ ), delocalized and nonergodic (energy density between $E_{S}$ and $E_{r}$ ), and delocalized and ergodic (energy density larger than $E_{r} \simeq E_{O}$ ). Note that an ergodic phase necessarily has delocalized states since they are essential for diffusive transport, which is a defining characteristic of ergodicity.

\section{ENERGY-RESOLVED dc CONDUCTIVITY}

The presence of $\delta\left(E_{n}-E_{m}\right)$ in Eq. (2) implies that states with essentially equal energies $\left(E_{n} \simeq E_{m}\right)$ (due to the broadening of the $\delta$ function) contribute $|\langle m|J| n\rangle|^{2}$ to $\sigma_{\mathrm{dc}}$. Therefore, one can study the contribution of the three phases, many body localized (region I), nonergodic and extended (region II), and ergodic (region III), separately from the dc conductivity:

$$
\sigma_{\mathrm{dc}} \simeq \sigma_{\mathrm{dc}, I}+\sigma_{\mathrm{dc}, I I}+\sigma_{\mathrm{dc}, I I I} .
$$

Figure 6 shows the variation of $\log _{10}\left(\sigma_{\mathrm{dc}, n}\right)$ as a function of the system size, where $n=I, I I, I I I$ denote the contribution from the three different regions for $v=1 / 4$. Here again, we average $\log _{10}\left(\sigma_{\mathrm{dc}, n}\right)$ over 96000,18000 , and 900 randomly chosen offset angles $\phi$ for system sizes 12,16 , and 20, respectively. As seen in Fig. $6 \sigma_{\mathrm{dc}, I}$ appears to decay much faster with system size compared to $\sigma_{\mathrm{dc}, I I}$ and $\sigma_{\mathrm{dc}, I I I}$. In contrast $\sigma_{\mathrm{dc}, I I}$ undergoes a slower decay with the system size, while $\sigma_{\mathrm{dc}, I I I}$ hardly decays at all with increasing system sizes. The diagnostics of the previous section show that region I has properties of the MBL phase, namely, Poisson distributed energy levels, area law scaling of the entanglement entropy, and ETH violation. It is known that in the MBL phase the dc conductivity decreases exponentially with increasing system size and goes to zero in the thermodynamic limit $[38,39,43]$. Therefore, the slower decay of $\sigma_{\mathrm{dc}, I I}$, which corresponds to the nonergodic delocalized phase, compared to that in region
I (the MBL phase), suggests that the nonergodic extended phase has a dc conductivity which in the thermodynamic limit goes to zero, but more slowly than in the MBL phase. This is perhaps indicative of subdiffusive transport (i.e., power law decay of conductivity with the system size) in the nonergodic delocalized phase. While, in principle, subdiffusive and localized transport would be best identified by a plot of the $\mathrm{dc}$ conductivity as a function of system size appearing to be linear on a log-log plot and a semilog plot, respectively, such behavior is not always seen in numerical studies on small system sizes [43]. However, to the extent that it is reasonably well established that transport due to MBL states does, indeed, give an exponentially decreasing conductivity with system size [38,39], the slower speed of decay of the dc conductivity with system size in the nonergodic extended phase seems consistent with subdiffusive transport.

\section{DISCUSSION AND CONCLUSIONS}

We have calculated the ac and dc conductivities (Figs. 1, 2, 3, and 4) and energy-resolved level spacing statistics [Figs. 5(a)-5(c)] to understand the effects of the nonergodic extended phase on transport. We identified the nonergodic (ETH violating, like the many-body localized eigenstates) yet extended (with volume law scaling of entanglement entropy, like the thermal eigenstates) eigenstates for different filling fractions (Fig. 5).

Looking at the energy-resolved level spacing, we find that the average value of the level spacing ratio $r_{n}$ changes as a function of energy density from the Poisson value $\left(\left\langle r_{n}\right\rangle_{\text {Poisson }} \simeq 0.386\right)$ to the GOE value $\left(\left\langle r_{n}\right\rangle_{\mathrm{GOE}} \simeq 0.5295\right)$ and that there is an energy density $E_{r}$ which separates these two regions. We also notice that the transition from Poissonian to GOE statistics is concurrent with the transition between the nonergodic and ergodic phases $\left(E_{O}\right)$ as identified by analyzing the validity of ETH as a function of energy density. The energy density $E_{s}$ corresponding to the transition between area law entangled eigenstates and volume law entangled eigenstates is less than $E_{O}$ or $E_{r}$ for all cases. Therefore, the energy level statistics captures the nonergodic to ergodic transition as opposed to the localized to delocalized transition as captured by entanglement entropy. This is consistent with what happens in other systems, for example, in the context of the Anderson model in the Bethe lattice $[47,48]$, where, for weak disorder, the system is in an extended and nonergodic phase and has Poisson level statistics.

In our studies of conductivity (Figs. 1 and 3), we have observed signatures of subdiffusive transport: (i) the exponent $\alpha$, obtained from the log-log plots of $\sigma(\omega)$ vs $\omega$, which lies between 0 and 1 , and (ii) vanishing dc conductivity, where the dc conductivity appears to go to zero in the thermodynamic limit slower than in the MBL phase, where the conductivity decreases exponentially with increasing system sizes. Studying the contributions of the three different kinds of eigenstates to the dc conductivity (Fig. 6), we observe that the contribution of the ergodic states is indicative of diffusive transport where it hardly decays with increasing system sizes. On the other hand, the conductivity in the nonergodic localized phase appears to decay much faster with increasing system sizes, while in the nonergodic extended phase it appears to decay at 

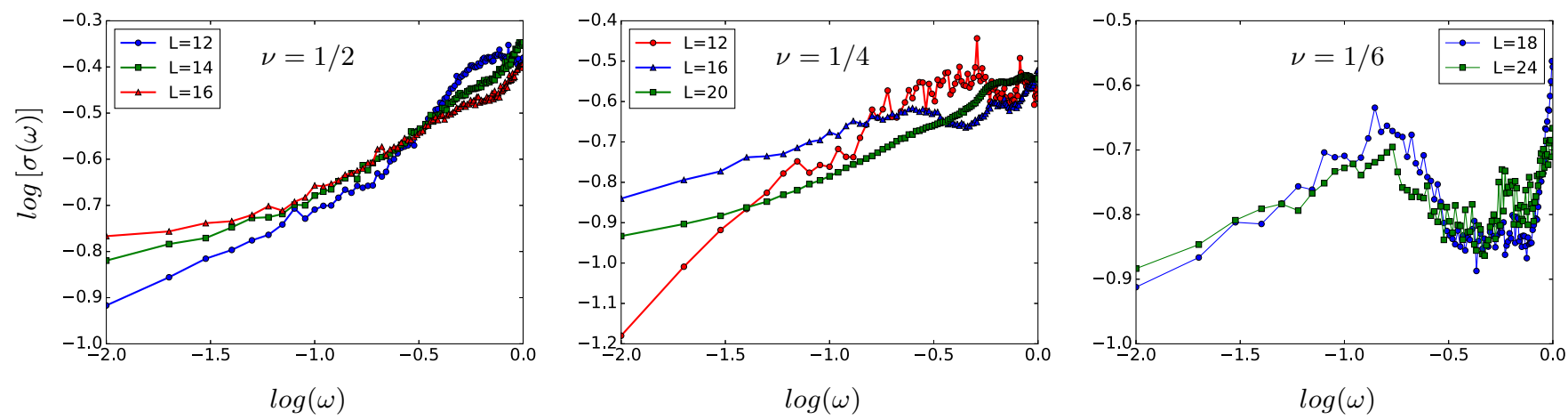

FIG. 7. The variation of $\sigma(\omega)$ vs $\omega$ for different system sizes at different filling fractions $v$. The width $\eta$ of the Lorentzian is chosen to be $0.001 \Delta$ for all cases, where $\Delta$ is the mean level spacing.

a rate in between the two cases. As the nonergodic localized phase (MBL phase) is expected to have an exponential decay of the dc conductivity with increasing system size, a slower decay of the dc conductivity contribution from the nonergodic extended phase is perhaps suggestive of a power law decay of the same with system size. A similar power law decay was also observed [43] for systems with random disorder where the sub-diffusive phase arises due to the Griffiths effect. We note that the signature of subdiffusive transport in the ac conductivity appears more pronounced in the dc conductivity, as can be seen from the discussion in Secs. II and IV. We thus provide evidence suggestive of subdiffusive transport even in the presence of a deterministic quasiperiodic potential which does not allow for Griffiths regions (which occur in the presence of random disorder), which we attribute to the presence of the nonergodic extended phase. A subdiffusive phase has also been reported in the interacting Aubry-André model near the critical region of the thermal-MBL transition [42] and in the Fibonacci model [44] in the presence of large interaction, while for small interaction the model shows only diffusive behavior. The Fibonacci model and the Aubry-André model also do not allow for the Griffiths scenario as rare regions of on-site potential do not appear. However, both these cases are slightly different than ours, in which we observe the subdiffusive transport in the presence of interaction with a strength equal to the hopping parameter and deep in the thermal region away from the thermal-MBL transition $\left(h_{c} \simeq 1.6 t\right)$ [32]. We do not investigate whether the microscopic pictures for the subdiffusive behavior of all these models are same.
In Ref. [49], the possibility of an alternative picture for the subdiffusive transport in a disordered $X X Z$ chain was presented in which the findings contradict the predictions of the Griffiths effect. Such an alternative picture, if found, might lead to a microscopic picture of subdiffusion in models irrespective of the presence of rare regions.

\section{ACKNOWLEDGMENTS}

S.M. thanks the Quantum information Science and Technology (QuST) initiative of the Department of Science and Technology (DST), government of India, for support.

\section{APPENDIX: SYSTEM SIZE DEPENDENCE OF $\sigma(\omega)$}

Figure 7 shows the system size dependence of $\sigma(\omega)$ versus $\omega$ curves at different filling fractions. Here we see the dependence of $\sigma(\omega)$ on $\omega$ is almost independent of system size for fillings $v=1 / 2$ and $1 / 6$; for $v=1 / 4$ there is a large dependence on the system size. While for $L=12, v=1 / 4$ the ac conductivity for the considered range of $\omega$ depends strongly on the choice of broadening $\eta$, the other two system sizes, $L=16,20$, do not have any $\eta$ dependence. Therefore, it is not clear why there is this system size dependence in the $\sigma(\omega)$ vs $\omega$ curves for $L=16$ and 20 . We also see that the same effect also occurs for the Aubry-André model $\left[h_{i}=\right.$ $h \cos (2 \pi \alpha i+\phi)]$ at filling $v=1 / 4$. This needs to be further investigated.
[1] P. W. Anderson, Phys. Rev. 109, 1492 (1958).

[2] D. Basko, I. Aleiner, and B. Altshuler, Ann. Phys. (NY) 321, 1126 (2006).

[3] V. Oganesyan and D. A. Huse, Phys. Rev. B 75, 155111 (2007).

[4] M. Žnidarič, T. Prosen, and P. Prelovšek, Phys. Rev. B 77, 064426 (2008).

[5] A. Pal and D. A. Huse, Phys. Rev. B 82, 174411 (2010).

[6] J. H. Bardarson, F. Pollmann, and J. E. Moore, Phys. Rev. Lett. 109, 017202 (2012).

[7] M. Serbyn, Z. Papić, and D. A. Abanin, Phys. Rev. Lett. 111, 127201 (2013).
[8] D. A. Huse, R. Nandkishore, and V. Oganesyan, Phys. Rev. B 90, 174202 (2014).

[9] J. Z. Imbrie, Phys. Rev. Lett. 117, 027201 (2016).

[10] M. Schreiber, S. S. Hodgman, P. Bordia, H. P. Lüschen, M. H. Fischer, R. Vosk, E. Altman, U. Schneider, and I. Bloch, Science 349, 842 (2015).

[11] T. Kohlert, S. Scherg, X. Li, H. P. Lüschen, S. Das Sarma, I. Bloch, and M. Aidelsburger, Phys. Rev. Lett. 122, 170403 (2019).

[12] J. M. Deutsch, Phys. Rev. A 43, 2046 (1991).

[13] M. Srednicki, Phys. Rev. E 50, 888 (1994). 
[14] M. Rigol, V. Dunjko, and M. Olshanii, Nature (London) 452 , 854 (2008).

[15] R. K. Panda, A. Scardicchio, M. Schulz, S. R. Taylor, and M. Žnidarič, Europhys. Lett. 128, 67003 (2020).

[16] S. Iyer, V. Oganesyan, G. Refael, and D. A. Huse, Phys. Rev. B 87, 134202 (2013).

[17] R. Modak, S. Mukerjee, E. A. Yuzbashyan, and B. S. Shastry, New J. Phys. 18, 033010 (2016).

[18] A. Chandran, I. H. Kim, G. Vidal, and D. A. Abanin, Phys. Rev. B 91, 085425 (2015).

[19] M. Rigol, Phys. Rev. Lett. 103, 100403 (2009).

[20] L. F. Santos and M. Rigol, Phys. Rev. E 81, 036206 (2010).

[21] R. Modak, S. Mukerjee, and S. Ramaswamy, Phys. Rev. B 90, 075152 (2014).

[22] R. Modak and S. Mukerjee, New J. Phys. 16, 093016 (2014).

[23] S. Aubry and G. André, Ann. Isr. Phys. Soc. 3, 18 (1980).

[24] J. Settino, N. W. Talarico, F. Cosco, F. Plastina, S. Maniscalco, and N. Lo Gullo, Phys. Rev. B 101, 144303 (2020).

[25] M. Griniasty and S. Fishman, Phys. Rev. Lett. 60, 1334 (1988).

[26] S. Das Sarma, S. He, and X. C. Xie, Phys. Rev. B 41, 5544 (1990).

[27] S. Ganeshan, J. H. Pixley, and S. Das Sarma, Phys. Rev. Lett. 114, 146601 (2015).

[28] N. Lo Gullo, C. V. Ambarish, T. Busch, L. Dell'Anna, and C. M. Chandrashekar, Phys. Rev. E 96, 012111 (2017).

[29] J. Settino, N. Lo Gullo, A. Sindona, J. Goold, and F. Plastina, Phys. Rev. A 95, 033605 (2017).

[30] R. Modak and S. Mukerjee, Phys. Rev. Lett. 115, 230401 (2015).

[31] R. Modak, S. Ghosh, and S. Mukerjee, Phys. Rev. B 97, 104204 (2018).

[32] X. Li, S. Ganeshan, J. H. Pixley, and S. Das Sarma, Phys. Rev. Lett. 115, 186601 (2015).
[33] D.-L. Deng, S. Ganeshan, X. Li, R. Modak, S. Mukerjee, and J. H. Pixley, Ann. Phys. (Berlin, Ger.) 529, 1600399 (2017).

[34] M. Pino, L. B. Ioffe, and B. L. Altshuler, Proc. Natl. Acad. Sci. USA 113, 536 (2016).

[35] J. Vidal, D. Mouhanna, and T. Giamarchi, Phys. Rev. Lett. 83, 3908 (1999).

[36] J. Vidal, D. Mouhanna, and T. Giamarchi, Phys. Rev. B 65, 014201 (2001).

[37] S. Mukerjee, V. Oganesyan, and D. Huse, Phys. Rev. B 73, 035113 (2006).

[38] T. C. Berkelbach and D. R. Reichman, Phys. Rev. B 81, 224429 (2010).

[39] O. S. Barišić and P. Prelovšek, Phys. Rev. B 82, 161106(R) (2010).

[40] K. Agarwal, S. Gopalakrishnan, M. Knap, M. Müller, and E. Demler, Phys. Rev. Lett. 114, 160401 (2015).

[41] M. Žnidarič, A. Scardicchio, and V. K. Varma, Phys. Rev. Lett. 117, 040601 (2016).

[42] Y. B. Lev, D. M. Kennes, C. Klöckner, D. R. Reichman, and C. Karrasch, Europhys. Lett. 119, 37003 (2017).

[43] F. Setiawan, D.-L. Deng, and J. H. Pixley, Phys. Rev. B 96, 104205 (2017).

[44] V. K. Varma and M. Žnidarič, Phys. Rev. B 100, 085105 (2019).

[45] S. Gopalakrishnan, M. Müller, V. Khemani, M. Knap, E. Demler, and D. A. Huse, Phys. Rev. B 92, 104202 (2015).

[46] A. Rényi, in Proceedings of the Fourth Berkeley Symposium on Mathematical Statistics and Probability (University of California Press, Berkeley, California, 1961), Vol. 1, pp. 547-561.

[47] A. De Luca, B. L. Altshuler, V. E. Kravtsov, and A. Scardicchio, Phys. Rev. Lett. 113, 046806 (2014).

[48] G. Biroli, A. C. Ribeiro-Teixeira, and M. Tarzia, arXiv:1211.7334.

[49] M. Schulz, S. R. Taylor, A. Scardicchio, and M. Žnidarič, J. Stat. Mech. (2020) 023107. 